\title{
Cervical Strip Biopsy for High-Grade Cervical Intraepithelial Lesions: a Valid Alternative to Conventional Punch Technique
}

\author{
Streifenbiopsie der Cervix uteri bei High Grade SIL: \\ eine Alternative zur konventionellen Knipsbiopsie
}

A. Schneider ${ }^{1}$, K. Wagner ${ }^{1}$, C. Rakozy ${ }^{1}$, C. Stolte ${ }^{2}$, P. Bothur-Schäfer ${ }^{3}$, T. Welcker ${ }^{3}$, N. Choly ${ }^{1}$, A. Roesgen ${ }^{1}$, H. Rothe ${ }^{3}$, G. Böhmer ${ }^{2}$

${ }^{1}$ Institute for Dysplasia and Cytology, Berlin

${ }^{2}$ Institute for Dysplasia and Cytology, Hannover

3 Institute for Dysplasia and Cytology, Göttingen
Key words

- high-grade SIL

- strip biopsy

$\checkmark$ painless

Schlüsselwörter

- High Grade SIL

- Streifenbiopsie

- schmerzlos

received 20.3.2015

revised $\quad 30.6 .2015$

accepted $\quad 5.7 .2015$

\section{Bibliography}

DOI http://dx.doi.org/ 10.1055/s-0035-1557816

Geburtsh Frauenheilk 2015; 75: 1063-1068 @ Georg Thieme

Verlag KG Stuttgart · New York ISSN 0016-5751

\section{Correspondence}

Achim Schneider MD MPH

Institute for Dysplasia

and Cytology

Hohenzollerndamm 123

14199 Berlin

schneider@izd-berlin.de

\section{Abstract}

$\nabla$

Introduction: To evaluate, if targeted strip biopsies decrease trauma/pain perception while maintaining diagnostic accuracy in patients with the diagnosis of high-grade squamous intraepithelial lesions of the uterine cervix.

Patients and Methods: Between July 1st and December 31st 2014 we performed colposcopically directed strip biopsies in 102 patients with colposcopic suspicion of high-grade squamous intraepithelial lesions of the uterine cervix. We used a $3 \mathrm{~mm}$ curette for harvesting tissue samples under VITOM $^{\circledR}$ videocolposcopy. So far, 60 patients underwent additional loop excision. Histologic examination of strip biopsies and loop specimens included routine hematoxylin and eosin staining as well as immunohistochemical staining for p16, Ki 67 and stathmin-1.

Results: 55 patients (53\%), were histologically diagnosed with cervical intraepithelial neoplasia grade 3 on strip biopsies. Adenocarcinoma in situ was diagnosed in 2 patients (2\%), cervical intraepithelial neoplasia grade 2 in 35 patients (34\%), and cervical intraepithelial neoplasia grade 1 in 10 patients (10\%). The agreement between histologic results of strip biopsy and loop specimen was highly significant: In all 60 strip biopsies diagnosed with high-grade squamous intraepithelial lesions this diagnosis was confirmed histologically during follow-up loop specimen excision (high-grade squamous intraepithelial lesions in 58 patients, invasive disease in 2 patients). The pain level experienced during strip biopsy was rated on average 0.25 on a scale from 0 to 10 . No clinically significant bleeding was reported.

Conclusion: Targeted strip biopsies with a $3 \mathrm{~mm}$ curette are a reliable procedure to diagnose highgrade squamous intraepithelial lesions of the uterine cervix and yield high patient satisfaction (๑ Video 1 ).

\section{Zusammenfassung \\ $\nabla$}

Fragestellung: Können bei Frauen mit V.a. High Grade squamous intraepithelial Lesions der Cervix uteri kolposkopisch gezielt entnommene Streifenbiopsien die Schmerzempfindung vermindern und trotzdem eine hohe diagnostische Sicherheit gewährleisten?

Patientinnen und Methoden: Zwischen 1. Juli und 31. Dezember 2014 führten wir bei $104 \mathrm{~Pa}-$ tientinnen mit kolposkopischem Verdacht auf High Grade squamous intraepithelial Lesions gezielte Streifenbiopsien durch. Kontrolliert durch VITOM $^{\circledR}$-Videokolposkopie benutzten wir eine 3-mm-Kürette zur Gewebegewinnung. Bei 60 dieser Patientinnen erfolgte im späteren Verlauf eine Schlingenexzision. Die histologische Untersuchung der Streifenbiopsien und Schlingenexzidate erfolgte mittels Hämatoxylin und Eosin sowie immunhistochemischer Färbung für p16, Ki 67 und stathmin-1.

Ergebnisse: Bei 55 Patientinnen (53\%) wurde in der Streifenbiopsie histologisch eine zervikale intraepitheliale Neoplasie Grad 3 diagnostiziert. Adenocarcinoma in situ wurde bei 2 Patientinnen (2\%), zervikale intraepitheliale Neoplasie Grad 2 bei 35 Patientinnen (34\%), und zervikale intraepitheliale Neoplasie Grad 1 bei 10 Patientinnen gefunden (10\%). Die Übereinstimmung der histologischen Ergebnisse zwischen Streifenbiopsie und Schlingenexzidat war exzellent: Alle 60 Streifenbiopsien mit der Diagnose High Grade squamous intraepithelial Lesions wurden am späteren Schlingenexzidat bestätigt (High Grade squamous intraepithelial Lesions bei 58 Patientinnen, Invasion bei 2 Patientinnen). Die Schmerzempfindung während und nach Streifenbiopsie betrug $0.25 \mathrm{im}$ Durchschnitt bei einer Skala zwischen 0 und 10. Keine klinisch auffällige Blutung wurde festgestellt.

Schlussfolgerung: Gezielte Streifenbiopsien mit einer 3-mm-Kürette bilden eine zuverlässige Me- 
thode zur Diagnose von High Grade squamous intraepithelial Lesions verbunden mit einer hohen Patientinnenzufriedenheit (๑ Video 2).

\section{Introduction}

The aim of secondary prevention of cervical cancer is the detection of women with high-grade squamous intraepithelial lesions of the uterine cervix (HSIL) or high-grade cervical intraepithelial neoplasia (CIN 2 and CIN 3) and treatment of CIN 3 and of CIN 2, where CIN 3 cannot be excluded.

Diagnosis of high-grade CIN requires histologic verification in tissue biopsies. Tissue is usually harvested by colposcopically directed punch biopsies. The majority of patients experience pain and bleeding with varying intensity ([1], http://www.cancerforums.net/threads/15812-Rough-Colposcopy-and-Cervical-biopsy-experience).

High trait anxiety prior to and post colposcopy is a risk factor for experiencing pain [3]. Topical application or local injection of anaesthetics increases the duration of the procedure with little or no effect on perception of pain [4-6]. Other techniques such as asking the patient to cough or offering the patient visual distraction during biopsy have limited effect $[5,7]$.

Therefore, the ideal biopsy technique should minimize trauma with no loss in diagnostic accuracy. An intrinsic feature of highgrade CIN is decreased adherence between epithelium and stroma. This gives rise to the colposcopic "rag sign" [8]. Neoplastic cervical epithelium scales off easily, even by slight contact with a swab or speculum and can be evaluated histologically. This phenomenon is passed down in personal communication by colposcopists and pathologists and formed also the basis of cold knife scraping of the portio as an alternative to conization or hysterectomy [2]. However, its biological basis remains unclear.

In our study we addressed the question if the trauma associated with conventional punch biopsy can be diminished or even annihilated by scraping off small strips of epithelium without causing discomfort to the patient. We correlated our colposcopic and histologic findings by strip biopsy and loop specimen with the patient's experience of trauma and pain prospectively.

\section{Patients and Methods \\ $\nabla$}

\section{Patient recruitment}

Between June 2014 and December 2014, 140 patients with a history of an abnormal Pap smear, high-risk HPV in a cervical smear, or abnormal clinical findings of the cervix uteri were referred to the Institute for Cytology and Dysplasia, Berlin, Germany. All patients referred to our colposcopy clinic were consecutively included in our study. Patients with colposcopic normal findings $(n=4)$, minor change $(n=19)$, transformation zone type 3 as defined by IFCPC [11], $(n=10)$, patients with visible invasive cancer $(n=1)$ and patients with a history of hysterectomy (3) or previous radiation (1) were not excluded from our study. Given these criteria 102 out of 140 patients (73\%) were eligible for our evaluation.

In our institute all patients undergoing video recorded procedures have to give written consent for archiving imaging material and agree to be consulted prospectively for their medical history and well-being during follow-up. The study protocol was not pre-

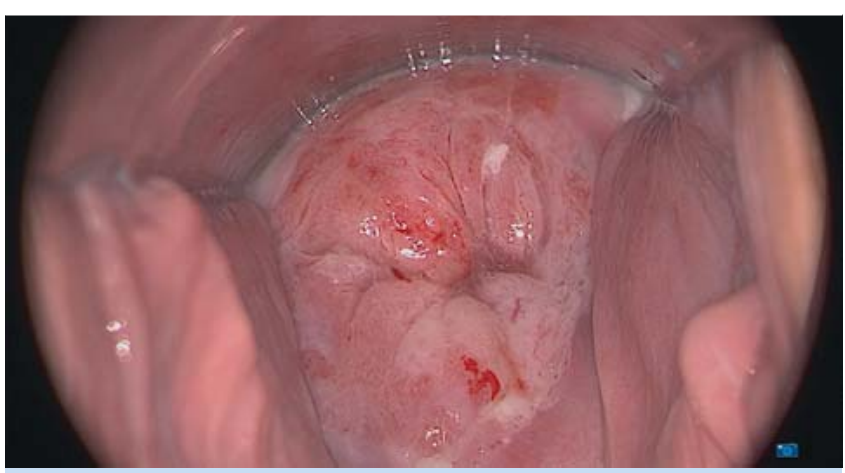

Fig. 1 An atypical transformation zone type 1 with opaque acetowhite epithelium and ridge sign at 5 and 6 o'clock can be identified in this 36year-old woman on oral contraceptives (Gravida 1, Para 1 ) with a history of a Pap smear with ASC-H and detection of high-risk HPV (not specified). All subsequent figures relate to this patient.

sented to an Internal Review Board since all procedures as well as all data collected were part of our clinical routine.

An elaborated workflow is established in our institute: all patients with suspicious findings are presented in an interdisciplinary conference where cytologists, gynecologists, and pathologists are present. Cytologic and histologic findings, FULL HD video exoscopy (colposcopy) and HPV test results are demonstrated.

\section{Colposcopy}

In our prospective study setting each patient underwent video exoscopy, using the VITOM ${ }^{\circledR}$ System. The VITOM ${ }^{\circledR}$ System, a video exoscope based system (KARL STORZ, Tuttlingen, Germany), allows FULL HD video documentation of the colposcopic examination. This technique is used for reevaluation and communication of colposcopic findings [9].

\section{Videocolposcope guided strip biopsy and loop excision} Histologic confirmation by VITOM ${ }^{\circledR}$ guided strip biopsy was performed on all patients with a colposcopic diagnosis of major change, suggestive of CIN 2 or CIN 3 ( $\bullet$ Figs. 1 to 5 , $\bullet$ Video 1 ). For colposcopic grading we mainly rely on 4 pathognomonic signs which we described previously [10]: the diagnosis of major change is made when inner border sign, ridge sign, rag sign and/ or cuffed crypt openings are seen. The presence of coarse mosaicism or punctuation were used as adjuncts in the diagnosis of major change.

After obtaining the histopathologic result of strip biopsy a followup Vitom guided loop excision was performed as a second procedure in all patients with a diagnosis of CIN 3 in strip biopsy, and in patients with a histological diagnosis of CIN 2 in strip biopsy, when older than 25 years with an atypical transformation-zone type 2 or type 3 or cytologic diagnosis suggestive of CIN 3.

\section{Histologic evaluation}

Histology of biopsies and loop excisions was performed on formalin-fixed, paraffin-embedded tissue, which was serially sectioned and stained with hematoxylin/eosin ( Fig. 6). All tissue 


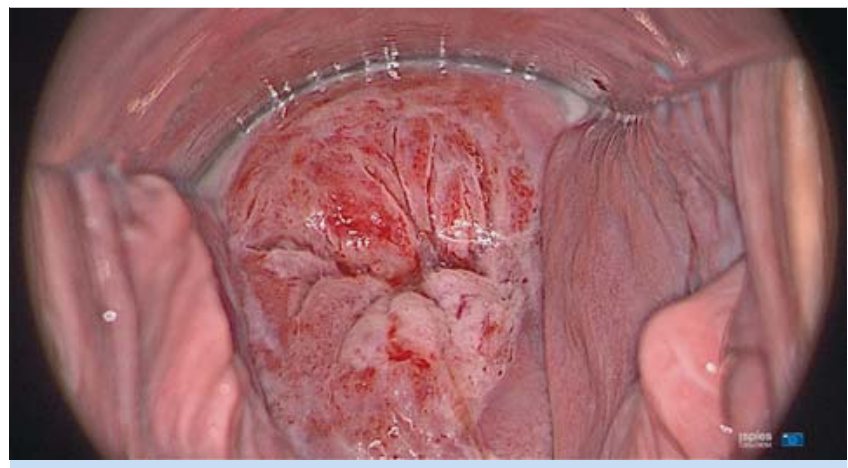

Fig. 2 Using the SPIES mode CLARA and CHROMA (Karl Storz Image 1 SPIESTM) contrast setting: two prominent ridges can be more clearly identified at 5 and 6 o'clock.

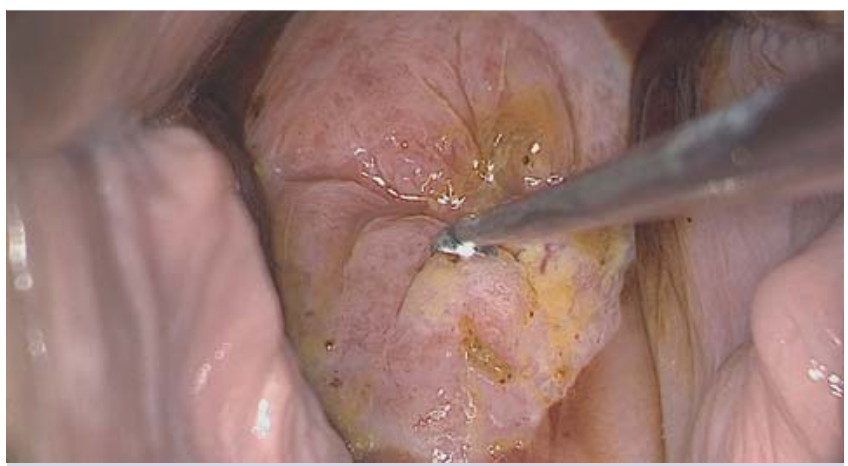

Fig. 4 A 3 mm curette (Fa. Krönauer, Tuttlingen, Germany) is targeted to an area with ridge sign at 6 o'clock.

samples underwent immunohistochemical staining according to standard protocols for possible detection of high-grade CIN using the following markers, p16, Ki 67 and stathmin-1 (STMN1) [1820]. These markers are especially useful when small tissue particles are analyzed. CIN was classified according to the criteria described by Crum [13] and WHO [14].

CIN 2 or CIN 3 expressed p16 and Ki67 and stathmin-1 into the middle or upper epithelial level ( $\bullet$ Figs. 7 to 9 ).

\section{Pain evaluation}

Patients were asked if they experienced discomfort or pain prior and directly after the strip biopsy. Pain was subjectively measured on a scale from 0 to 10 with 0 equaling no pain and 10 experiencing excruciating pain [12].

- Level 0: no pain

1-3: mild pain (nagging, annoying, interfering little with ADLs [Activity of Daily Living])

- 4-6: moderate pain (interferes significantly with ADLs)

- 7-10: severe pain (disabling; unable to perform ADLs)

Patients' pain impression was documented in our data base.

\section{Statistical analysis}

Accordance between the histo-pathologic results of strip biopsy and subsequent loop excision was analysed by binomial test.

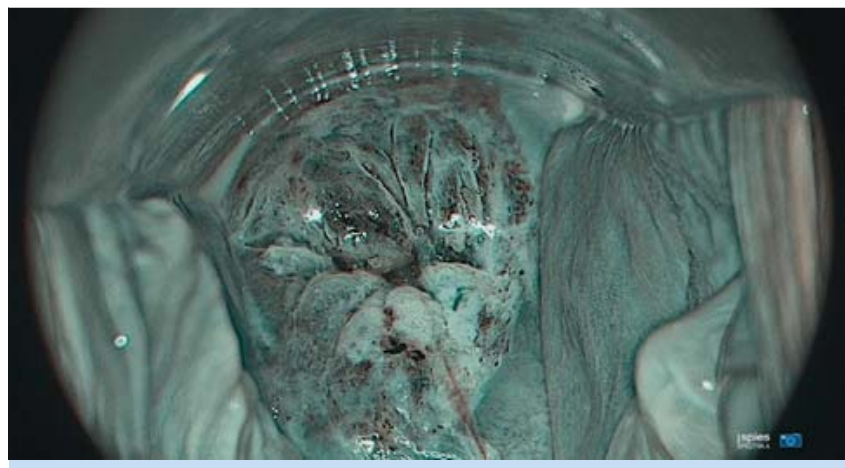

Fig. 3 Using the SPIES mode SPECTRA A color setting: two prominent ridges can be more clearly identified at 5 and 6 o'clock.

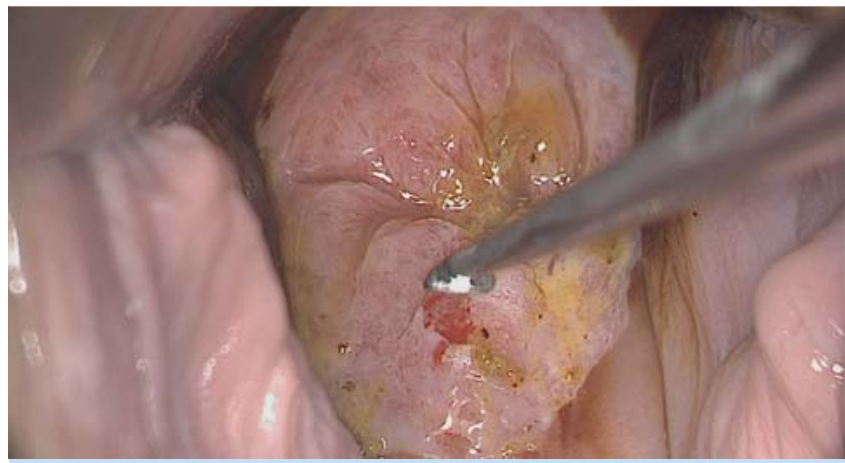

Fig. 5 A small strip of tissue has been mobilized at 6 o'clock leaving a small erosion. Tissue is subsequently harvested with the curette or an atraumatic forceps.

\section{Results}

\section{$\nabla$}

\section{Study population}

Between June 2014 and December 2014, 102 patients with a history of an abnormal Pap smear, high-risk HPV in a cervical smear, or abnormal clinical findings of the cervix uteri were included in our study. The average age was 34.1 years and all patients underwent cervical strip biopsy. A follow-up loop excision was per-

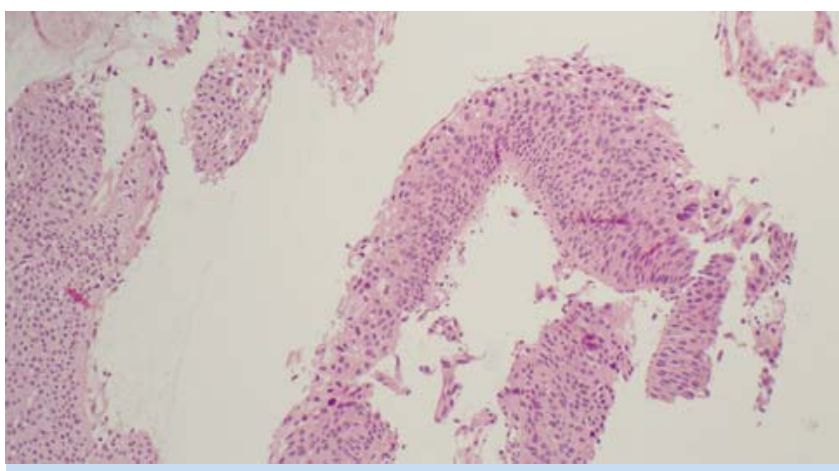

Fig. $6 \mathrm{H} \& \mathrm{E}$ staining of the strip specimen shows non-keratinizing epithelium lacking differentiation throughout the epithelial layers and hyperchromasia and anisonucleosis consistent with CIN 2 and CIN 3. 
formed in 60 patients. 32 patients were current smokers, 10 patients previous smokers and 60 patients had never smoked. 90 patients were premenopausal, 8 patients perimenopausal and 3 patients postmenopausal.

On cervical strip biopsy, $54 \%(n=55)$ of patients were diagnosed with CIN 3, $2 \%(n=2)$ with adenocarcinoma in situ (ACIS), $34 \%$ $(\mathrm{n}=35)$ with CIN 2 and 10\% $(\mathrm{n}=10)$ with CIN1 (ه Table 1$)$.

\section{Accordance of histopathological results}

of strip biopsy and loop specimens

95\% of CIN 3 diagnosed by strip biopsy were confirmed in the loop specimen ( Table 2). Statistically the proportion of CIN 3 in loop excision was at least 95\% and of CIN 2 or CIN $3100 \%$. CIN 2 and microinvasion were diagnosed in loop specimen in one patient, respectively.

$47 \%(n=9)$ of CIN 2 diagnosed by strip biopsy showed CIN 2 in the loop specimen; 53\% ( $n=10)$ of biopsies were diagnosed as CIN 3. Strip biopsies with diagnosis of CIN 2 were significantly associated with CIN 2 or CIN 3 in loop excision. In these 10 patients who had been underdiagnosed by strip biopsy five showed only small areas of CIN 3 in the loop specimen (horizontal extension less than $1 \mathrm{~mm}$ ). No patient showed less than CIN 2 in the loop specimen. In both patients with the histopathologic diagnosis ACIS in strip biopsy ACIS was confirmed in the loop specimen with additional microinvasion in one of these patients.

\section{Quality of obtained tissue by strip biopsy}

for histopathological evaluation

The adequacy of the biopsy was judged to be sufficient for a reliable diagnosis in 95 of 102 (93.1\%) specimens by the pathologist. The remaining cases had not sufficient material for a definite grading of CIN.

Histologically the strips of epithelium were usually free of underlying stroma and showed high expression of p16 and Ki67 and stathmin-1 up to the middle or upper third layer depending on the grade of CIN ( $\bullet$ Figs. 7 and 8). Immunostaining was of adequate quality in all samples independent of tissue size or CIN grade. Especially in small tissue particles immunostaining was valuable in order to identify HSIL.

Discrimination between CIN 2 and CIN 3 was impossible, when the epithelial strips had been oriented horizontally: a horizontal section through the intermediate epithelial layer where all cells expressed p16 and Ki67 was diagnosed as CIN 2 which lead to undercall of 5 strip biopsies where the loop specimen showed CIN 3 ( Table 2 ).

\section{Pain evaluation}

82 of 102 patients (80.4\%) reported no sensation during strip biopsy, 15 patients (14.7\%) reported a pain score 1 and 5 patients a pain score 2 (4.9\%). The mean pain score was 0.25 . There was no association between pain score and age, grade of CIN or smoking. None of our patients reported significant bleeding following strip biopsy. None of our patients needed a second examination or intervention.

\section{Discussion \\ $\nabla$}

In a colposcopy clinic the colposcopist identifies areas suggestive of CIN 2, CIN 3 or invasive cancer. These areas are biopsied and histologically evaluated. If invasive disease is suspected a punch biopsy or loop excision including epithelium and stroma is man-
Table 1 Histological results of strip biopsies performed in 102 patients.

\begin{tabular}{|lc|}
\hline Total number & $102(100)$ \\
\hline CIN 1 & $10(9.8)$ \\
\hline CIN 2 & $35(34.3)$ \\
\hline CIN 3 & $55(53.9)$ \\
\hline ACIS & $2(2)$ \\
\hline
\end{tabular}

Table 2 Correlation of histological results between strip biopsies and followup loop excision in 60 patients.

\begin{tabular}{|c|c|c|}
\hline & Loop excision & \\
\hline Strip biopsy & 60 & \\
\hline \multirow[t]{3}{*}{ CIN 2, $n=19(100)$} & CIN 1 & 0 \\
\hline & CIN 2 & $9(47.4)$ \\
\hline & CIN 3 & $10(52.6)$ \\
\hline \multirow[t]{4}{*}{$\mathrm{CIN} 3, \mathrm{n}=39(100)$} & CIN1 & 0 \\
\hline & CIN 2 & $1(2.6)$ \\
\hline & CIN 3 & $37(94.8)$ \\
\hline & Microinvasion & $1(2.6)$ \\
\hline \multirow[t]{2}{*}{$\operatorname{ACIS} 2, \mathrm{n}=2(100)$} & ACIS & $1(50)$ \\
\hline & Adeno CA & $1(50)$ \\
\hline
\end{tabular}

datory for a correct diagnosis. However, colposcopy clinics are rarely visited by women with invasive disease and the main task is to identify patients with high-grade SIL. This was also the case in our series where 2 patients showed microinvasion which could only be confirmed in the loop specimen and may have been missed by punch biopsy. $93 \%$ of the strip biopsies were of adequate size and quality for proper histopathologic evaluation which is in accordance with international guidelines (i.e. more than $90 \%$ ) [15].

Thus, we showed that the use of targeted small strip biopsies give an excellent and highly significant correlation with the underlying preneoplastic disease when the area with the most severe histologic changes is properly identified by colposcopy.

In a metaanalysis of 7873 paired punch/definitive histology comparison sensitivity for a punch biopsy to diagnose CIN 2+ disease is $91.3 \%$ (95\% CI $85.3-94.9 \%$ ) and specificity is $24.6 \%$ (95\% CI $16.0-35.9 \%$ ) [16]. The sensitivity we had using previously defined pathognomonic signs was $90.2 \%, 92$ out of 102 patients with major change (i.e. $90.2 \%$ had CIN 2+) and, thus, comparable to punch biopsy results. Since we biopsied exclusively patients with major change we cannot calculate specificity in this study. In our study the correlation between the strip result and definite histology was $97 \%$ for prediction of CIN 3 or microinvasion. CIN 2 on strip biopsy was upgraded to CIN 3 in $53 \%$ of patients on definite histology. This was partly due to small foci of CIN 3 within CIN 2 and partly due to horizontal orientation of the epithelium in the histologic evaluation. Since all patients with histologically confirmed diagnosis of CIN 2 and age older than 25 years underwent loop excision this "undercalling" of CIN 3 was no disadvantage for the patient.

Biopsy trauma may be minimized with a superficial small diameter biopsy forceps. Still, even if this procedure is performed by an experienced colposcopist, $53 \%$ of women reported pain, $46 \%$ discharge, $79 \%$ post-biopsy bleeding and $43 \%$ some change to their subsequent menstrual bleeding; the authors conclude that cervical punch biopsies carry a substantial risk of side-effects, similar to LLETZ [1]. 


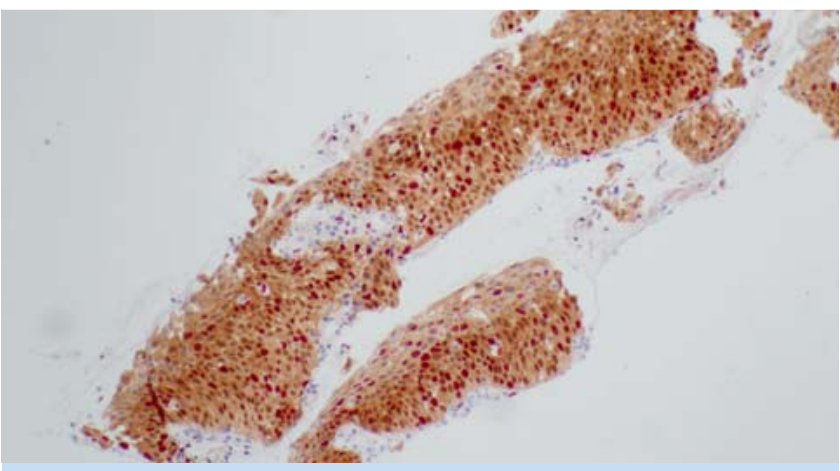

Fig. 7 Immuno-staining of the strip specimen using p16 and Ki67 antibodies shows expression of both antigens in the upper layer of the epithelium consistent with CIN 3.

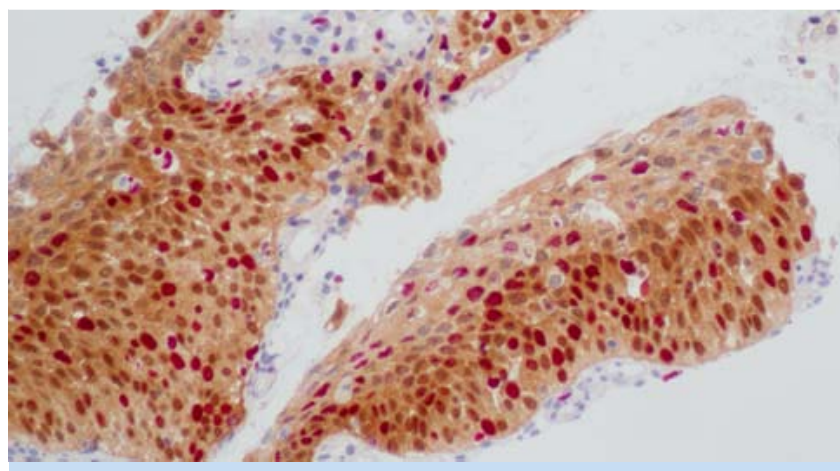

Fig. 8 Immuno-staining using p16 and Ki67 antibodies showing anisonucleosis and increased nuclear-cytoplasmic ratio consistent with CIN 3 at higher magnification compared to $\bullet$ Fig. 7.
Post-colposcopy distress is correlated with pain experienced during colposcopy. Women with high trait anxiety have a greater risk for developing negative psychosocial consequences after the procedure [3].

In order to alleviate pain within colposcopically directed cervical punch biopsies, the topical application of $10 \%$ lidocaine spray was compared to placebo in a randomized, double-blind study in 214 women: pain scores were $2.18 \pm 1.7$ in the lidocaine group and $2.31 \pm 1.6$ in the placebo group and, thus, patients did not benefit from topical lidocaine anesthesia prior to cervical punch biopsy or ECC [4].

Injected local anaesthetics were not more effective than forced coughing during biopsy as shown in a prospective study of 100 consecutive patients; they added pain related to cervical injection and increased operative time significantly [5].

In another randomized controlled study of 114 patients the pain score obtained during the first cervical biopsy was significantly lower when local anesthesia was applied, but the injection also added to the duration of the procedure. It was concluded that local anesthesia, but not forced coughing, provides significant pain relief during cervical biopsy [6].

Visual distraction may also be used to reduce pain associated with colposcopically directed biopsies. This was shown in a nonrandomized intervention design where women were distracted by images on a light diffuser installed within the examination room's ceiling light: these women had a 54\% reduction of postexamination pain compared with women who did not receive the visual distraction [7].

Gentle language is another method to effectively decrease pain associated with surgical procedures: A randomized study in patients with cervical biopsies compared standard language to gentle, non-pain-based language. The results showed a pain score of 3.1 versus 2.9 , which was not significantly different [17].

So far no successful strategy has been defined to help alleviate biopsy trauma for women visiting a colposcopy clinic. Therefore, innovative concepts need to be investigated. We believe that strip biopsies are a viable option for improving the quality of life for these women.

This study has several strengths: First, we prospectively evaluated a cohort of consecutive patients in a routine setting where the team included several colposcopists and pathologists.

Second, we used previously defined pathognomonic signs which have been analysed over a 20 -year period. This allowed for a tight

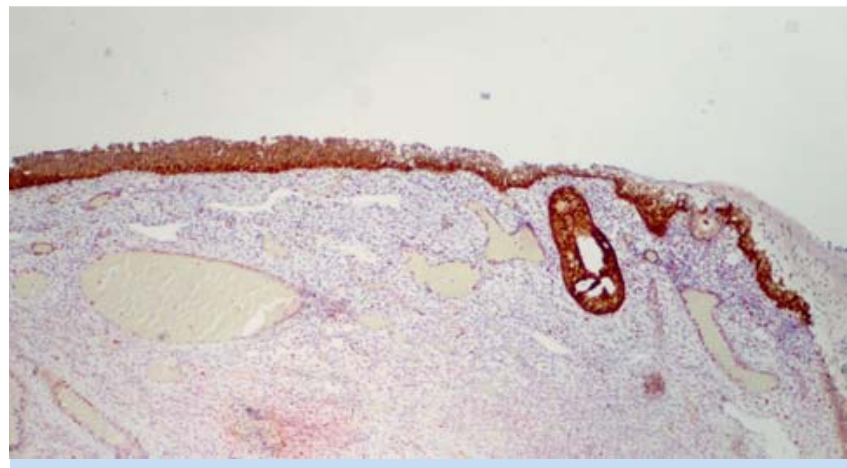

Fig. 9 Definite histological evaluation of the loop specimen reveals CIN 3 on immunostaining using p16 and Ki67 antibodies with glandular expansion and transition to normal non-keratinizing epithelium at the right side.

correlation between colposcopic findings and histologic outcome.

Third, no random sampling was done. Our patients were treated with targeted biopsies. This method decreased fear and increased cooperation.

Fourth, our small strip biopsies left the main area of CIN intact. There was no tissue destruction by random/deep biopsies and no significant subsequent inflammatory reaction. This facilitated a definite evaluation in loop excisions.

However, there are a few limitations to this study:

First, the pathologists were not blinded to the results of the strip biopsy when evaluating the loop specimen for definite histological diagnosis.

Second, for paraffin embedding orientation of the small epithelial specimen of strip biopsy needs diligence and training. If oriented horizontally, tangential sections will result. Pathologists see only basal and parabasal layer and may have to undercall CIN 3 since the upper epithelial layers are not demonstrated.

Third, our institution routinely performs immunhistochemical stains for p16, Ki 67 and STMN1 on cervical specimen. This is not part of the currently recommended routine work-up and is not supported by guidelines. However, immunohistochemistry is an extremely useful adjunct when analyzing minute strips of tissue. Therefore, our immunoresults are probably difficult to reproduce in a routine setting when only $\mathrm{H} \& \mathrm{E}$ stains are performed (albeit on usually larger biopsy material). 
Fourth, we did not perform loop excisions on 10 patients with CIN1 (these women are still under follow-up). This verification bias may have increased the accuracy of our results.

\section{Conclusion \\ $\nabla$}

We feel that strip biopsies are a painless and valid technique for histopathologic diagnosis of high-grade SIL which eventually increases the benefit for the respective patient. To further evaluate our findings, our next step will be a prospective study comparing the accuracy between strip and punch biopsies, and their influence on the quality of life of women who underwent these procedures. We will try to determine whether strip biopsies are viable to be integrated into routine colposcopy.

\section{Financial Disclosure}

$\nabla$

Achim Schneider acts as advisor for Karl Storz. Until September 2013 he received honoraria for lectures and support for training of Fellows in Gynecologic Oncology. All authors have contributed to the manuscript. The Institute for Cytology and Dysplasia received instruments and equipment as a loan from Karl Storz $\mathrm{GmbH}$, Tuttlingen. No funding was received for this study.

\section{Acknowledgment}

$\nabla$

The authors thank Carla Schneider for reviewing the manuscript. The authors thank Stefanie Kroll for the excellent technical assistance in preparing the manuscript.

\section{Conflict of Interest \\ $\nabla$}

None.

\section{Videos 1 and 2}

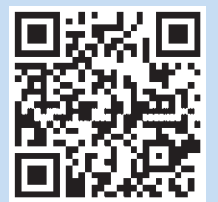

The English and German version of this videoclip can be found online:

http://dx.doi.org/10.1055/s-0035-1557816

\section{References}

1 The TOMBOLA (Trial Of Management of Borderline and Other Low-grade Abnormal smears) Group; Sharp L, Cotton S, Cochran C et al. After-effects reported by women following colposcopy, cervical biopsies and LLETZ: results from the TOMBOLA trial. BJOG 2009; 116: 1506-1514

2 Egger H, Hommel G, Michalzik K. Portio Abschabung und Cervix-Curettage eine Alternative zur Konisation bei positiver Zytologie. Arch Gynecol 1979; 227: 249-265

3 Kola-Palmer S, Walsh JC. Correlates of psychological distress immediately following colposcopy. Psychooncology 2015; 24: 819-824

4 Öz M, Korkmaz E, Cetinkaya $N$ et al. Comparison of topical lidocaine spray with placebo for pain relief in colposcopic procedures: a randomized, placebo-controlled, double-blind study. J Low Genit Tract Dis 2015; 19: 212-214

5 Bogani G, Serati M, Cromi A et al. Local anesthetic versus forced coughing at colposcopic-guided biopsy: a prospective study. Eur J Obstet Gynecol Reprod Biol 2014; 181: 15-19

6 Naki MM, Api O, Acioglu HC et al. Analgesic efficacy of forced coughing versus local anesthesia during cervical punch biopsy. Gynecol Obstet Invest 2011; 72: 5-9

7 Carwile JL, Feldman S, Johnson NR. Use of a simple visual distraction to reduce pain and anxiety in patients undergoing colposcopy. J Low Genit Tract Dis 2014; 18 : $317-321$

8 Vercellino GF, Erdemoglu E, Chiantera Vet al. Validity of the colposcopic criteria inner border, ridge sign and rag sign for detection of high-grade cervical intraepithelial neoplasia. Obstet Gynecol 2013; 121: 624-631

9 Vercellino GF, Erdemoglu E, Kyeyamwa S et al. Evaluation of the VITOM in digital high-definition video exocolposcopy. J Low Genit Tract Dis 2011; 15: 292-295

10 Vercellino GF, Erdemoglu E, Chiantera Vet al. Clinical relevance of objectifying colposcopy. Arch Gynecol Obstet 2015; 291: 907-915

11 Bornstein J, Bentley J, Bosze P et al. 2011 Colposcopic Terminology of the International Federation for Cervical Pathology and Colposcopy. Obstet Gynecol 2012; 120: 166-172

12 Huskisson EC. Measurement of pain. J Rheumatol 1982; 9: 768-769

13 Crum CP. Papillomavirus-related Changes and premalignant and malignant squamous Lesions of the uterine Cervix. In: Clement PB, Young $\mathrm{RH}$, eds. Tumors and tumorlike Lesions of the uterine Corpus and Cervix. New York: Churchill Livingstone; 1993: 51-83

14 Kurman R, Carcangiu ML, Herrington S, Young R, eds. WHO Classification of Tumours of female reproductive Organs. 4th ed. Lyon: IARC; 2014

15 Luesley D, Leeson S, eds. Colposcopy and programme management. Guidelines for the NHS Cervical Screening Programme. 2nd ed. Sheffield: NHS Cancer Screening Programmes Fulwood House; 2010

16 Underwood M, Arbyn M, Parry-Smith W et al. Accuracy of colposcopydirected punch biopsies: a systematic review and meta-analysis. BJOG 2012; 119: 1293-1301

17 Dalton M, Dangel A, Strohsnitter W et al. The impact of gentle language on pain perception during colposcopy: a randomized controlled trial. J Low Genit Tract Dis 2014; 18: 314-316

18 Howitt BE, Nucci MR, Drapkin R et al. Stathmin-1 expression as a complement to p16 helps identify high-grade cervical intraepithelial neoplasia with increased specificity. Am J Surg Pathol 2013; 37: 89-97

19 Reuschenbach M, Wentzensen N, Dijkstra MG et al. p16INK4a immunohistochemistry in cervical biopsy specimens: a systematic review and meta-analysis of the interobserver agreement. Am J Clin Pathol 2014; 142: 767-772

20 Bergeron C, Ordi J, Schmidt D et al.; European CINtec Histology Study Group. Conjunctive p 16INK4a testing significantly increases accuracy in diagnosing high-grade cervical intraepithelial neoplasia. Am J Clin Pathol 2010; 133: 395-406 